\title{
Damage in Polymer Bonded Energetic Composites: Effect of Loading Rate
}

\author{
Rachael L. Boddy ${ }^{1} \cdot$ Peter J. Gould ${ }^{2}$ Andrew P. Jardine ${ }^{1} \cdot$ David M. Williamson $^{1}$
}

Received: 30 October 2015/ Accepted: 18 January 2016/Published online: 1 February 2016

(C) The Author(s) 2016. This article is published with open access at Springerlink.com

\begin{abstract}
Particulate composites are widely used in the materials world. An understanding of their damage behaviour under a variety of loading conditions is necessary to inform models of their response to external stimuli. In the present experimental study, fine and coarse grained RDXHTPB composites have been used to investigate the effect of loading rate on the degree of damage produced in polymer bonded explosives subjected to varying degrees of uniaxial compression. High strain rate loading $(4 \times$ $10^{3} \mathrm{~s}^{-1}$ ) was achieved using a direct impact Hopkinson pressure bar and low strain rate loading $\left(1 \times 10^{-2} \mathrm{~s}^{-1}\right)$ using an Instron mechanical testing machine. The causal metrics are the degree to which the samples were strained and the mechanical energy expended in straining them. The damage metric is the residual low rate compressive modulus of the samples. The quantitative, physically based, results discussed in terms of the Porter-Gould activated debonding damage model clearly demonstrate that for both fine and coarse fills there is a marked reduction in residual moduli as a function of imposed strain, and substantially less specific energy is required to cause the same level of damage at the lower strain-rate. In the case of the coarse grained composite there is some evidence for a change in damage mechanism at the higher strain-rate. We obtain a value for the measured work of adhesion and a measure of the effective modulus local to the damage site, as damage is actually occurring. The observed underlying behaviour should be broadly applicable to particulate composites,
\end{abstract}

David M. Williamson

dmw28@cam.ac.uk

1 SMF group, Cavendish Laboratory, University of Cambridge, Cambridge, UK

2 QinetiQ, Bristol, UK whenever stiff filler particles are held in a viscoelastic matrix.

Keywords Damage $\cdot$ Composite $\cdot$ Polymer bonded explosives $\cdot$ Dynamic deformation

\section{Introduction}

Particulate composites are widely used in the materials world, and in particular polymer bonded particulate composites are one of the most common types of consolidated energetic material. Such materials are typically composed of crystalline organic energetic filler particles bound in a plasticized polymeric binder system. They are intended to initiate only in response to an intentional stimulus, however, when these materials are subjected to mechanical insult, the micromechanical changes which occur within them (damage) have been shown to increase their sensitivity to further stimuli, and thus the likelihood of unintended reaction [1,2]. Furthermore, any reaction that does occur is likely to be more violent in nature [3, 4]. Gaining a thorough understanding of the nature of mechanical damage, and the factors which influence its extent, is therefore of great importance in ensuring the safe usage of such materials.

Above the glass transition condition of the polymer matrix, an important damage mechanism in polymer bonded explosives (PBXs) has been identified as debonding of filler particles from the binder system [5-7]; it is specifically this process which is addressed in the current study. Broadly, damage (debonding) in PBXs can be modelled either with regard to the mesoscale, with explicit representation of the microstructure and by prescribing locally varying properties [8-10], or else by a continuum representation. The former approach is necessarily computationally intensive and 
therefore, for the sake of simplicity in the current experimental study, we adopt the latter approach. In particular, we make use of the physically based Porter-Gould damage model which was especially developed to facilitate predictive modelling of such composites [11] by describing the effect of damage evolution on the elastic modulus of a PBX in terms of debonding. The basis for the model is the continuum damage mechanics result [12] that the residual Young's modulus, $E_{R}$, can be expected to degrade from the initial modulus, $E_{0}$, as a function of a damage parameter $D$ $(0 \leq D \leq 1)$ as

$E_{R}(D)=(1-D) E_{0 .}$

Specifically, the Porter-Gould damage model assumes that the mechanical energy delivered during a damage event acts to successively debond filler particles at an increasing (but finite) number of damage sites which are distributed homogeneously throughout the material, and hence to reduce the stiffness of the composite. Thus $D$ takes on a meaning which relates to the probability of a particle being debonded, a process described by a simple Arrhenius relation comparing the specific mechanical energy imparted to the sample, $w$, to a damage activation energy term, $w_{0}$, given by

$E_{R}(w)=\left(E_{0}-E_{L}\right)\left[1-\exp \left(-\frac{w_{o}}{w}\right)\right]+E_{L}$,

where $E_{L}$ describes a limiting modulus. Physically $E_{L}$ corresponds to the state where all the filler particles which can debond have done so, and reinforcement is at a minimum, but the sample retains some residual (limiting) stiffness.

The term $w_{o}$ can be associated with a Griffith type energy balance between the change in stored strain energy, the cost of destroying interface, and the work done in doing so [13]. A common conclusion in consideration of the debonding problem [14-16] is that

$w_{0} \propto \frac{\gamma(\dot{\varepsilon})}{r}$,

where $r$ is the radius of the filler particle and $\gamma(\dot{\varepsilon})$ is the measured surface energy required to destroy the interface. The term $\gamma(\dot{\varepsilon})$ is found to be equal to the thermodynamic work of adhesion magnified by the viscous loss in the binder which, above the glass transition condition, increases with strain rate $\dot{\varepsilon}$ [17-19]. Equation (2) can be equivalently re-cast on the basis of strain $\varepsilon$, making use of the identity $w=E \varepsilon^{2}$,

$E_{R}(\varepsilon)=\left(E_{0}-E_{L}\right)\left[1-\exp \left(-\frac{\varepsilon_{0}^{2}}{\varepsilon^{2}}\right)\right]+E_{L}$,

where $\varepsilon_{0}$ takes on the meaning of an activation strain, as per the study of Siviour et al. [16].

It follows from Eqs. (2) and (3) that we expect that the character of damage should dependent on strain-rate. Some

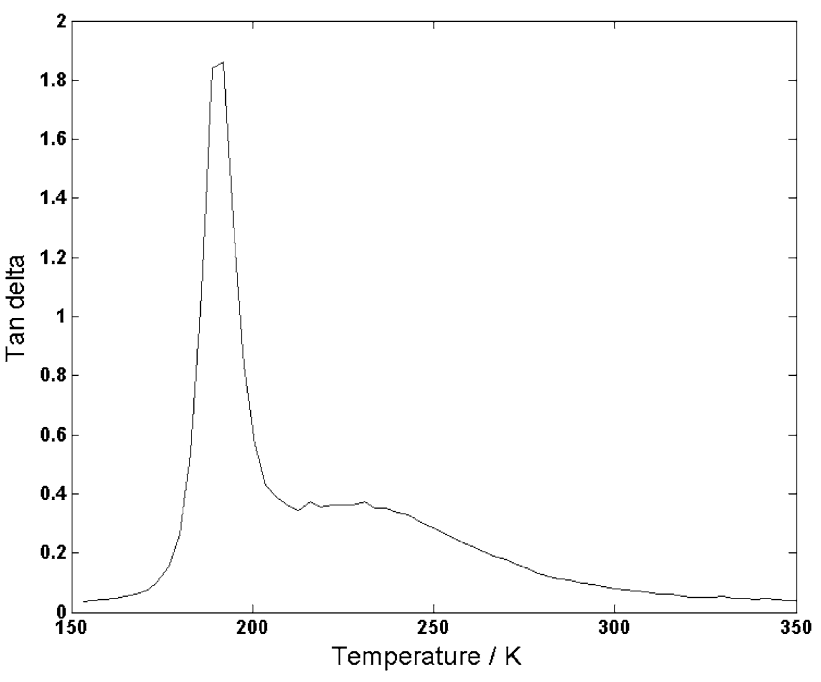

Fig. 1 Loss tangent of the HTPB binder measured by DMA at $1 \mathrm{~Hz}$. The value of $\tan (\delta)$ rises from 0.09 to 0.15 as temperature is decreased from 295 to $275 \mathrm{~K}$. On the basis of time-temperature superposition, and an equivalence factor of $4 \mathrm{~K}$ per decade of strain rate [20], it is reasonable to expect an equivalent increase in $\tan (\delta)$ at room temperature between the strain rates of $1 \times 10^{-2}$ to $4 \times 10^{3} \mathrm{~s}^{-1}$ used in the current study

insight into the expected behaviour can be obtained by considering the loss tangent of the HTPB binder used in the current study, obtained by dynamic mechanical analysis (DMA) at $1 \mathrm{~Hz}$ as a function of temperature, which is shown in Fig. 1. The data shows that viscous loss, as quantified by $\tan (\delta)$ at low rates and room temperatures is small. A generally applicable result regarding time-temperature superposition for polymers is that in relation to the glass transition event, a decade increase in strain rate can be considered approximately equivalent to a $4 \mathrm{~K}$ reduction in temperature [20]. In the present study, we inflict damage at room temperature and at a low and a high strain rate, separated by five orders of magnitude $\left(1 \times 10^{-2}\right.$ and $\left.4 \times 10^{3} \mathrm{~s}^{-1}\right)$, which can therefore be considered to be approximately equivalent to a $20 \mathrm{~K}$ reduction in temperature (or vice versa). Taken from Fig. 1, $\tan (\delta)$ approximately doubles in value when the temperature is reduced from 295 to $275 \mathrm{~K}$ (the small difference in strain rates between a DMA test at $1 \mathrm{~Hz}$ and $0.1 \%$ amplitude and a uniaxial test at $1 \times 10^{-2} \mathrm{~s}^{-1}$ can be ignored). Therefore, in relative terms, it is reasonable to expect that the loss factor might approximately double over the range of strain rates used in the present study $\left(1 \times 10^{-2}\right.$ to $\left.4 \times 10^{3} \mathrm{~s}^{-1}\right)$. However, in absolute terms, the loss remains small (only increasing from approximately 0.09$0.15)$. A result of the above is that we should expect a positive correlation between $\tan (\delta)$ and $\gamma(\dot{\varepsilon})$ (e.g. as reported by Charrault et al. [21]) and $w_{0}$. However, at the present time the precise mapping is not well understood.

The present study was motivated by examining the above arguments: 
Table 1 Constitution of the HTPB binder

\begin{tabular}{ll}
\hline HTPB constituents & $\%$ by mass \\
\hline HTPB (uncured polymer) & 60.3 \\
DOS (plasticizer) & 32.9 \\
IPDI (curing agent) & 5.6 \\
Lecithin (wetting agent) & 0.8 \\
Lowinox (antioxidant) & 0.4 \\
\hline
\end{tabular}

- Equations (2) and (4) suggest the residual modulus should fall as more mechanical energy is deposited into the samples (or equivalently the more they are strained).

- Equations (2) and (3) and the data of Fig. 1 suggest a positive correlation between $\dot{\varepsilon}$ and $w_{0}$, and therefore for a fixed composition the amount of energy required to cause a certain degree of damage should be greater at higher strain rates.

- Equation (3) suggests we should expect a negative correlation between and $r$ and $w_{0}$, and therefore for a fixed strain rate composites with coarse filler particles should damage more readily than those with fine fills.

Whilst the above arguments suggest trends, they are not quantitatively predictive. In particular, the magnitude of the effect of strain rate on the damage mechanism(s) is not currently well understood. To this end, we report experiments on two RDX-HTPB composites with different, nominally monomodal, particle size distributions. These samples have been initially compressed (damaged) either at a low $\left(1 \times 10^{-2} \mathrm{~s}^{-1}\right)$ or a high $\left(4 \times 10^{3} \mathrm{~s}^{-1}\right)$ strain rate, and then re-tested at the low strain-rate and their residual compressive moduli measured. The resulting residual moduli are fitted using the Porter-Gould damage model to provide a framework for further discussion.

\section{Experimental Method}

The two RDX-HTPB (cyclotrimethylenetrinitramine-hydroxyl-terminated polybutadiene) sample types used in this study were produced at QinetiQ Fort Halstead. QRX214 has a 0.60 volume fraction of fine RDX particles which can be approximated by a normal distribution of mean $43 \mu \mathrm{m}$ and standard deviation $31 \mu \mathrm{m}$. QRX217 has a 0.54 volume fraction of coarse RDX particles which can be approximated by a log-normal distribution of mean $275 \mu \mathrm{m}$ and standard deviation $125 \mu \mathrm{m}$. The HTPB binder is constituted as shown in Table 1. The theoretical densities of the two material types are 1484 and $1432 \mathrm{~kg} \mathrm{~m}^{-3}$ for QRX214 and QRX217 respectively. Cylindrical samples, $5 \mathrm{~mm}$ in diameter, were punched from $5 \mathrm{~mm}$ thick sheets. The dimensions of each sample were measured on an optical comparator to an accuracy of $\pm 0.05 \mathrm{~mm}$ before any mechanical testing. All the experiments described were performed at ambient room temperature $\sim 294 \mathrm{~K}$.

Low rate damage loading was performed on a commercial screw-driven Instron mechanical testing machine (model 5500); samples were compressed at a rate of $1 \times 10^{-2} \mathrm{~s}^{-1}$ over a range of different strains up to a maximum of approximately 0.6 . High rate damage loading was achieved using a direct impact Hopkinson pressure bar (DIHPB), which was preferred over a split Hopkinson pressure bar (SHPB) arrangement because of the higher sample strains which can be practicably achieved. The system was comprised of duralumin alloy bars of 0.5 inch diameter. The striker bar, impact bar and momentum trap were $0.1,1.0$, and $0.3 \mathrm{~m}$ length respectively. A pair of semiconductor strain gauges (Kulite semiconductor products AFP 500-090; gauge length $1.524 \mathrm{~mm}$; temporal resolution mounted on duralumin $310 \mathrm{~ns}$ ) were mounted at diametrically opposing positions (to eliminate any spurious signal which may be caused by unwanted bending modes) at the mid-point of the impact bar, to capture information about the deformation of the samples. The change in resistance of the gauges due to strain (gauge factor $\sim 140$ ) was converted to a voltage measurable with an oscilloscope (Tektronix TDS540) using a potential divider; full details of the circuit are given in Siviour [22].

In a conventionally instrumented direct impact system, such as the one described in the current study, the only wave to be considered is the one transmitted through the sample. As there is no information about the force at the front of the sample, stress equilibrium between the front and back faces must be assumed in order to obtain an expression for the engineering stress $\sigma$ in the sample a time $t$, which is given by

$\sigma(t)=\frac{F_{T}(t)}{A_{0}}$

where $F_{T}$ is the transmitted force obtained from the calibrated strain gauges and $A_{O}$ is the cross-sectional area of the sample. The strain of the sample at time $t$ is dependent on the velocity of the striker bar at that point in time. An expression for the length of the sample at a time after impact by the striker bar, $l(t)$, was originally published by Pope and Field [23] and is given by

$l(t)=l_{0}-v_{0} t+\frac{2}{z_{b}} \int_{0}^{t} F_{T}\left(t^{\prime}\right) d t^{\prime}$,

where $l_{O}$ is the original length of the sample, $v_{O}$ is the velocity of the striker bar immediately prior to impact (accurately measured using a laser interruption scheme) and $z_{b}$ is the impedance of the bar material. Equation (6) is only valid until the reflected wave from the rear of the striker bar reaches the specimen; for this reason and because of the need for relative large sample strains, a 
striker bar velocity of approximately $20 \mathrm{~m} \mathrm{~s}^{-1}$ was used. The engineering strain in the sample, $\varepsilon$, is simply given by

$\varepsilon(t)=1-\frac{l(t)}{l_{0}}$.

In the above analysis we have made use of the unphysical assumption of instantaneous stress equilibrium within the sample. In practice a commonly accepted approximation is that a minimum of three stress wave reverberations within the sample are required before equilibrium is achieved [24]. For the $5 \mathrm{~mm}$ length specimens used in the current study, struck at $20 \mathrm{~m} \mathrm{~s}^{-1}$, the samples would have only accumulated approximately $3 \%$ strain in that time. This figure is supported by a very recent study conducted by members of our research group at the Cavendish Laboratory; Lea and Jardine [25] make use of a direct impact bar instrumented with photon Doppler velocimetry (PDV) which additionally allows for the stress waves in the striker bar to be measured. Such information is not accessible using strain-gauges and the conventional one-wave approach of the current study. Their results indicate (for soft metal samples at least) that the conventional one-wave analysis rapidly approaches their twowave analysis (the greatest difference is below a strain of approximately $2 \%$, where the one-wave analysis consistently under predicts the sample stress) which in turn is well matched to conventional SHPB data analysed using the standard three-wave method. In terms of the work done on the samples, which is the main quantity of interest here (integral of stress-strain), over the strain range 0-0.6, their conventional one-wave and more rigorous two-wave data differ by less than $2 \%$. At the time the data of the current study were collected the two-wave experimental approach had not yet been developed, and whilst we might anticipate polymer (or PBX) samples might reach equilibrium slightly later (due to their lower mechanical impedances), the differences are not expected to significantly affect the conclusions of the current study.

In order to physically limit the strain in the samples damaged using the DIHPB approach, duralumin collars of specific lengths were placed around the samples; once the target strain was reached the collars prevented any further deformation by the initial loading pulse, or reloading by any subsequent reverberations within the bars. The inner diameters of the collars were sufficiently large that they did not impede radial expansion of the samples, collar lengths were chosen to permit a range of strains up to a maximum value of approximately 0.6 , as per the low rate damage samples.

The strains to which the samples were taken, and the energy expended in reaching those strains (calculated by numerically integrating the stress-strains curves) form our two causal damage metrics. The low-rate residual modulus was chosen as a measure of the damaged state. The choice was informed by the results reported by Drodge and Williamson [26], which showed a clear distinction between damage measured post factum by 'active' techniques such as moduli measurements via uniaxial compression or dynamic mechanical analysis (DMA), and damage measured by 'passive' techniques such as a measure of density, thermal conductivity, (or tomography). The 'active' techniques were found to be much more sensitive indicators of the presence of damage than the 'passive' techniques. It was concluded that the increased sensitivity is fundamentally due to crack re-closure at debond sites; a phenomenon which is most likely to be relevant to cross-linked binder systems such as HTPB which are largely elastic in nature. Such closed cracks cannot support tensile loads (a measureable loss of stiffness in the composite results), but will otherwise not contribute to a loss of density or connectivity (little to no measureable reduction in density or thermal conduction). The contrast between 'active' and 'passive' detection techniques was found to be most acute where fine filler particles were present.

Post-damage loading, the dimensions were re-measured, and the samples were re-tested on the Instron at the low rate of $1 \times 10^{-2} \mathrm{~s}^{-1}$, parallel to the original loading direction, this being a more practical choice than evaluation by DMA given the sample geometry. A residual modulus was measured for each sample from the initial linear part of the stress-strain curve, which was recorded as the damage metric. A detailed description of the experimental procedure is also given by Boddy [27]. In all cases the samples were lubricated using paraffin wax, which has previously been shown to result in very low friction coefficients when used with polymer samples [28].

In the context of discussing the results we use the term 'causal metrics' to describe the measured quantities that stimulate damage (in this study, the degree to which the samples were strained and the mechanical energy expended in straining them) and the term 'damage metrics' to describe the measured quantities that characterise damage (in this study, the residual low rate compressive modulus of the samples when re-tested parallel to the original loading direction).

\section{Results and Discussion}

Figure 2 shows the pristine response of the two material types at the low and high strain rates used in this study, and demonstrates the greater stiffness of the composites at the higher rate of strain. Figures 3 and 4 show the evolution of the compressive stress-strain response of the materials in response to increasing levels of damage inflicted at the lower strain rate applied using the Instron screw driven mechanical testing machine, and Figs. 5 and 6 at the higher 


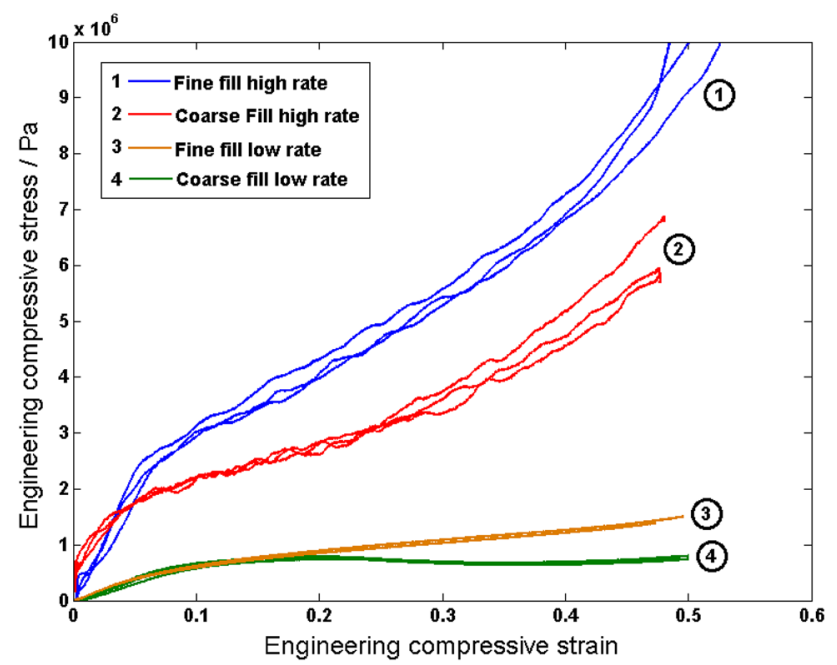

Fig. 2 Engineering stress-strain response of the pristine composites at the two strain rates of interest, $4 \times 10^{3} \mathrm{~s}^{-1}$ (upper curves) and $1 \times 10^{-2} \mathrm{~s}^{-1}$ (lower curves). Data obtained using a direct impact Hopkinson pressure bar apparatus and an Instron screw driven mechanical testing machine respectively. Three repeat measurements are shown for each situation

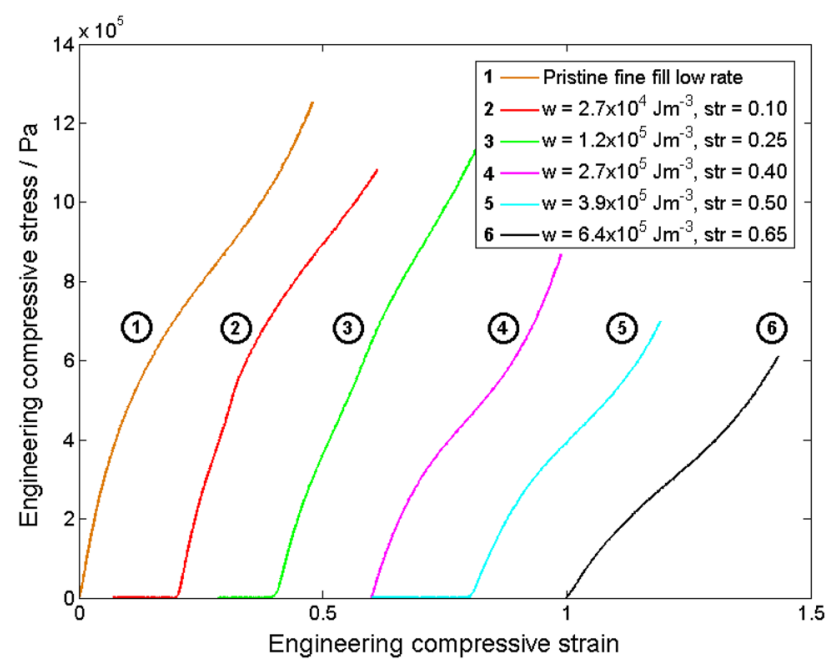

Fig. 3 Low rate compressive response of fine particle loaded QRX 214 following increasingly larger levels of damage inflicted at the lower rate of strain. Each curve has been offset by 0.2 strain for clarity. The energy input (w) and maximum strain (str) that the samples experienced during the damage phase are shown in the legend. The initial gradient (modulus) can be seen to decrease with increasing damage

strain rate using the direct impact Hopkinson pressure bar apparatus.

It is clear from Figs. 3, 4, 5, and 6 that the residual low rate moduli (initial linear portion of the stress-strain curves) decrease with increasing levels of damage, and that their use as a damage metric is justified. There are other signatures in the stress-strain responses that become progressively washed out with increasing damage; most notably the pristine coarse

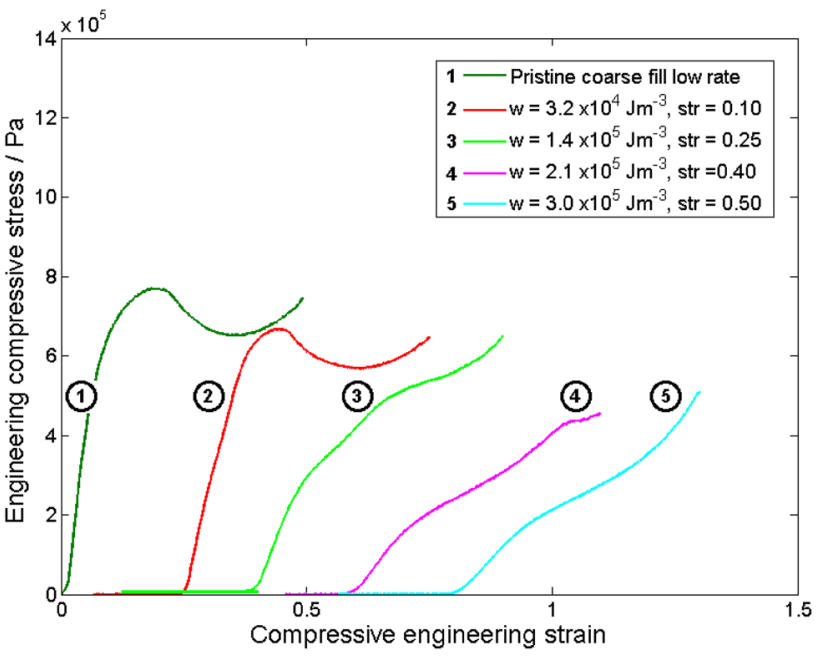

Fig. 4 Low rate compressive response of coarse particle loaded QRX 217 following increasingly larger levels of damage inflicted at the lower rate of strain. Each curve has been offset by 0.2 strain for clarity. The energy input (w) and maximum strain (str) that the samples experienced during the damage phase are shown in the legend. The initial gradient (modulus) can be seen to decrease with increasing damage

filled composite has a pronounced local maximum at a strain of approximately 0.18 and the fine loaded composite similarly has a less pronounced inflection point at approximately a strain of 0.25 . These 'large strain' features are related to damage and a loss of structural integrity, but the forms of these features are more complex, and their precise attribution is not obvious, and so are they not considered further in the present study.

Using data of the form shown in Figs. 3, 4, 5, 6, 7, and 8 show the normalised residual moduli as a function of the mechanical energy input to the samples in achieving the imposed strains when damaging the samples at either the low or the high rate. Figures 9 and 10 show the same normalised residual moduli as functions of imposed strain. Figures 7, 8, 9, and 10 clearly show that the residual moduli fall as more mechanical energy is deposited into the samples (or equivalently the more they are strained). It is also clear that under the same loading conditions, the composite with the coarse filler particles damages more readily than that with the fine fill.

The fits to the data shown in Figs. 7, 8, 9, and 10 use the Porter-Gould model as per Eqs. (2) and (4), normalised by the moduli of the undamaged material. The fits were calculated simultaneously using a Bayesian approach, allowing $w_{0}$ and $\varepsilon_{0}$ to be free parameters, but insisting that $E_{L}$ be common to both the energy and strain based descriptions. The coefficients for the optimal fits (which were found to be unique) are given in Table 2. In general terms, there is good agreement between the data and the fitted models, but there is a tendency for the models to over-predict the residual 


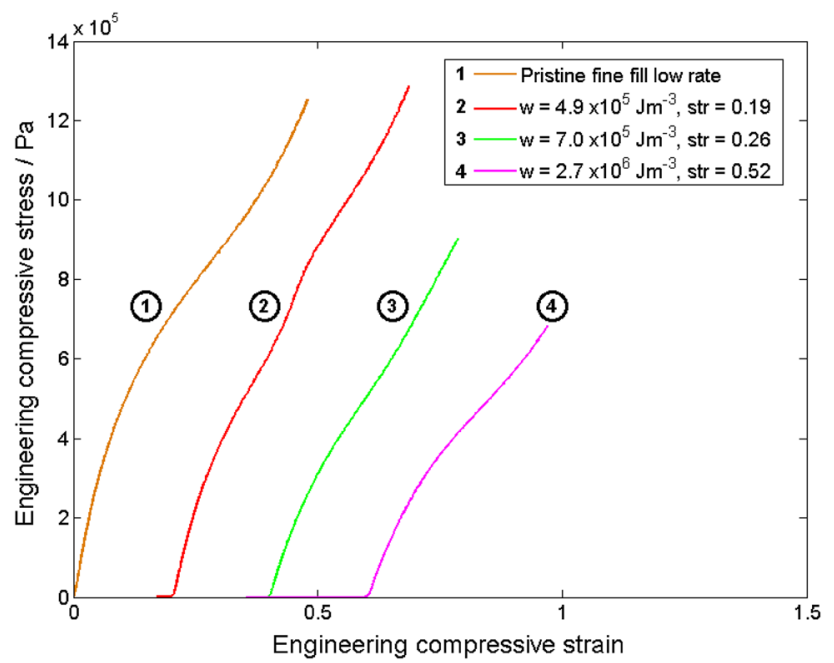

Fig. 5 Low rate compressive response of fine particle loaded QRX 214 following increasingly larger levels of damage inflicted at the higher rate of strain. Each curve has been offset by 0.2 strain for clarity. The energy input (w) and maximum strain (str) that the samples experienced during the damage phase are shown in the legend. The initial gradient (modulus) can be seen to decrease with increasing damage

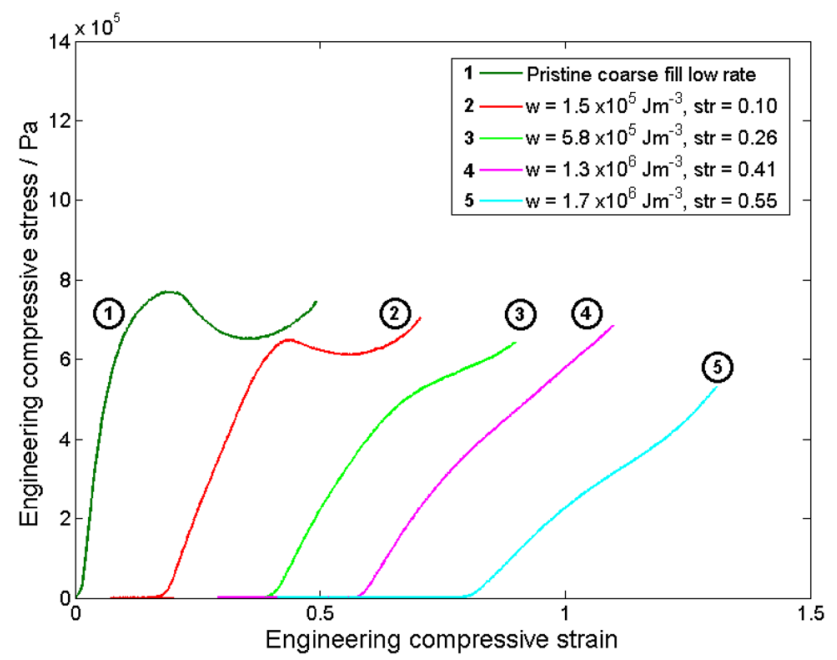

Fig. 6 Low rate compressive response of coarse particle loaded QRX 217 following increasingly larger levels of damage inflicted at the higher rate of strain. Each curve has been offset by 0.2 strain for clarity. The energy input (w) and maximum strain (str) that the samples experienced during the damage phase are shown in the legend. The initial gradient (modulus) can be seen to decrease with increasing damage

moduli at low levels of damage (particularly evident for the fine fill, low rate, data as a function of strain, shown in Fig. 9) and to also over-predict the residual moduli for high levels of damage (particularly evident for the coarse fill, low rate data as a function of specific energy, shown in Fig. 8). Possible reasons might be that $w_{0}$ and $\varepsilon_{0}$ are not constant, but may in fact vary with damage level, or that there are

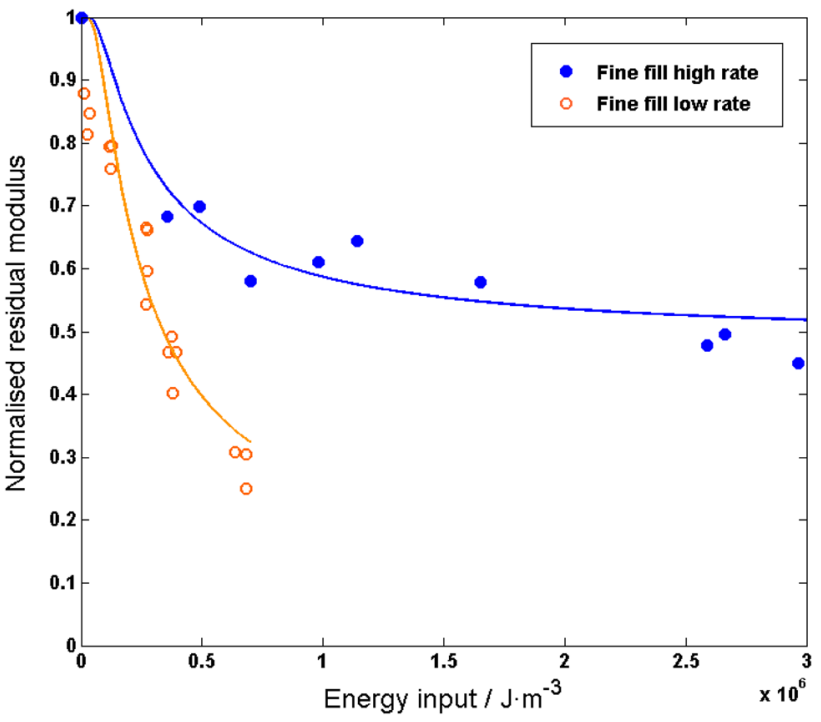

Fig. 7 Normalised residual low rate moduli for the fine loaded composite QRX214 as a function of specific energy input during deformation at the high and low rates. The fits to the data are the Porter-Gould model as per Eq. (2) normalised by the initial modulus. The error on the data points is approximately $5 \%$

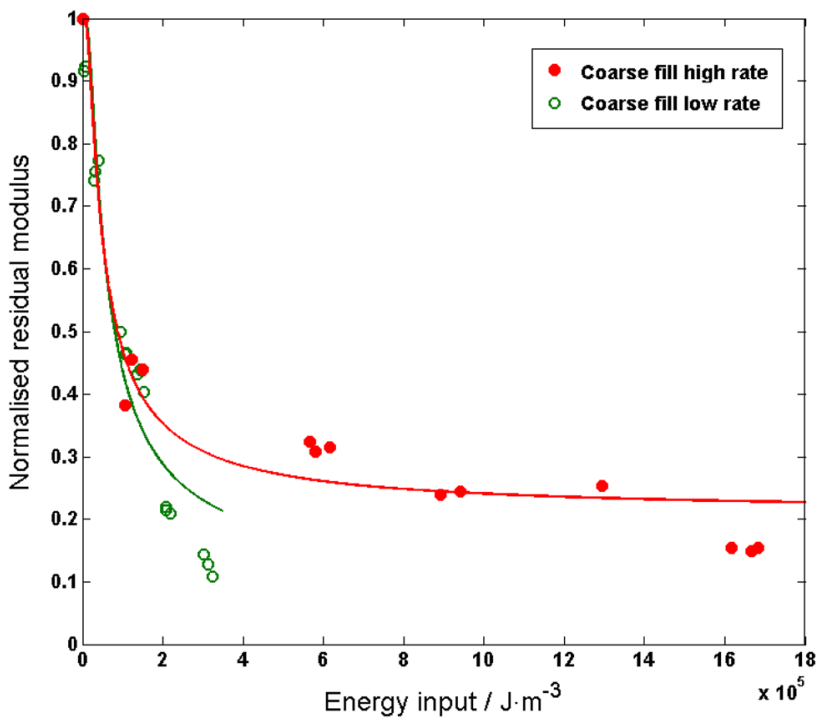

Fig. 8 Normalised residual low rate moduli for the coarse loaded composite QRX217 as a function of specific energy input during deformation at the high and low rates. The fits to the data are the Porter-Gould model as per Eq. (2) normalised by the initial modulus. The error on the data points is approximately $5 \%$

additional damage mechanisms operative which have a comparable magnitude to the effect of debonding.

A surprising observation which can be made for both materials is that the limiting moduli $E_{L}$, representing the residual modulus for the fully damaged state, appears to be greater when the damage is inflicted at the higher strain rate. We would expect the limiting moduli to represent the 


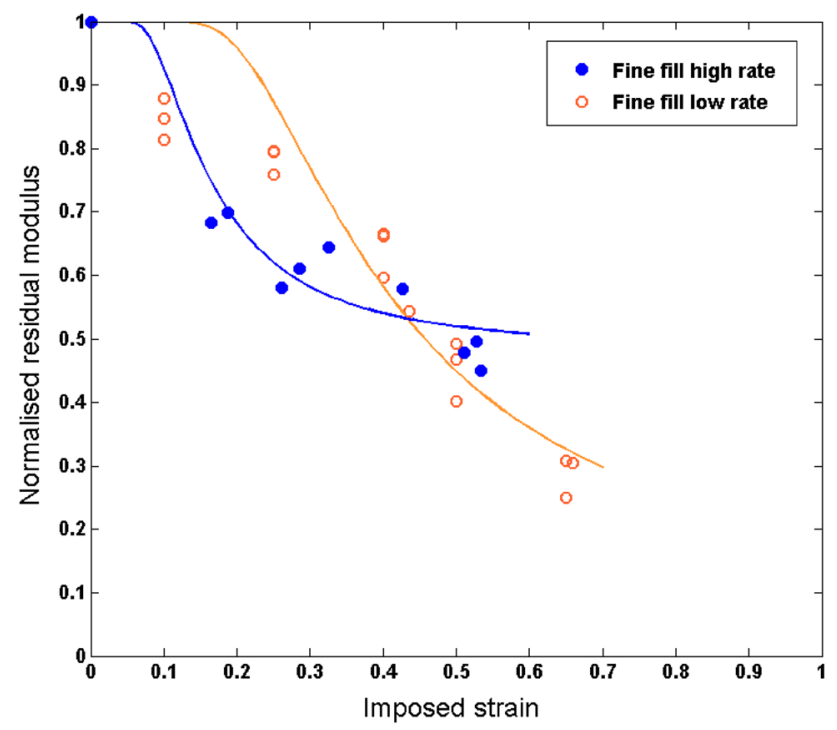

Fig. 9 Normalised residual low rate moduli for the fine loaded composite QRX214 as a function of imposed strain during deformation at the high and low rates. The fits to the data are the Porter-Gould model as per Eq. (4) normalised by the initial modulus. The error on the data points is approximately $5 \%$

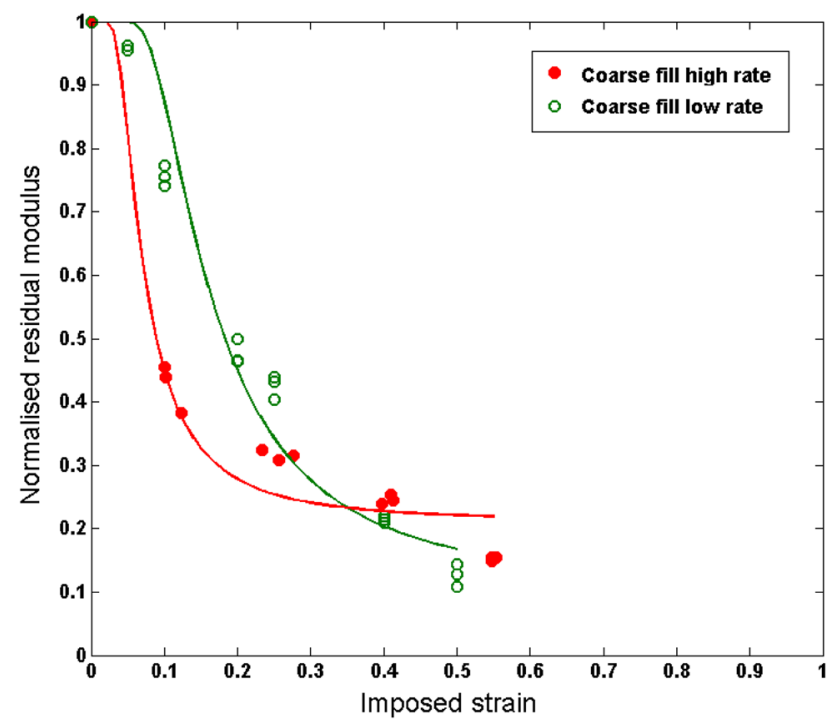

Fig. 10 Normalised residual low rate moduli for the coarse loaded composite QRX217 as a function of imposed strain during deformation at the high and low rates. The fits to the data are the Porter-Gould model as per Eq. (4) normalised by the initial modulus. The error on the data points is approximately $5 \%$

condition of the samples when all the particles have become debonded (in the limit of large energy expended on the samples), a process we expect to be independent of the strain rate at which damage occurs. There are (at least) two plausible explanations:

- Within the stated physical model, the interpretation is that there is a fraction of particles which cannot be debonded, regardless of how much damage is applied, and that fraction is greater at higher strain rates. The effect could be related to the increased levels of inertial confinement that the samples see at higher strain rates, since hydrostatic confinement is known to supress damage formation [29].

- Outside of the model as stated, is the possibility that at low strain rates there are additional damage mechanisms operative, which have a comparable magnitude of effect to debonding. One such candidate could be rupture of the binder between debonding sites, although this is normally considered energetically unfavourable [5].

Figures 7 and 8 and the data of Table 2 clearly show that for both fine and coarse loaded materials the mechanical energy required to produce a particular level of damage is dependent on the rate at which the sample is loaded. At the lower strain-rate substantially less specific energy is required to inflict the same level of damage. In the case of fine loaded QRX214, this can be attributed to both an increased damage activation energy, and a reduced limiting modulus. However, for coarse loaded QRX217, the cause is the reduced limiting modulus alone, and we note that the activation energy has actually decreased with loading rate. This observation in turn leads to a lower than expected resilience to damage at the higher strain rate.

Considered as a function of imposed strain, Figs. 9 and 10 , the fits to the data corresponding to damage at high and low rates are not so easily distinguished; the fits cross at a strain of approximately $0.4-0.5$, and there is considerable overlap of data points over much of the strain range. A consequence of the cross-over and overlap is an apparent insensitivity to strain-rate when using imposed strain as a causal metric for damage.

In addition to discussion of the fitted quantities, there are two further parameters which may be inferred. The first is the value of $\gamma(\dot{\varepsilon})$ which can be calculated from the analysis of Nicholson [14], by noting that for an incompressible material (which is a reasonable approximation for many rubbers) the constant of proportionality required to convert Eq. (3) into an equality is $\frac{24}{9}$. The calculated values of $\gamma(\dot{\varepsilon})$ are given in Table 2 . We note that for both material types the value of $\gamma(\dot{\varepsilon})$ is of order a few $\mathrm{J} \mathrm{m}^{-2}$ and this measured work of adhesion is approximately two orders of magnitude greater than the so-called thermodynamic values, which are usually of order a few tens of $\mathrm{mJ} \mathrm{m}^{-2}$. The observation is in keeping with our experience of adhesion experiments [30] and can be explained by the magnifying effect of viscous loss, as discussed above. In the case of the fine loaded composite the value of $\gamma(\dot{\varepsilon})$ increases with strain rate, in accordance with our understanding of the relationship to, and behaviour of, $\tan (\delta)$. However the rise in $\gamma(\dot{\varepsilon})$ is a relatively modest $\sim 20 \%$ compared to the $\sim 70 \%$ increase in 
Table 2 Coefficients of the Porter-Gould damage model fits to the data of Figs. 2, 3, 4, and 5 as per Eqs. (2) and (4) normalised by the initial moduli. The fits were calculated simultaneously using a Bayesian approach allowing $w_{o}$ and $\varepsilon_{o}$ to be varied freely but insisting that $E_{L}$ be common to both the energy and strain based descriptions.
The coefficients for the optimal fits were found to be unique. Also given are the initial moduli for the (physically) small samples used in the current study and inferred quantities $E_{d a m}$ and $\gamma(\dot{\varepsilon})$ which are the effective moduli during the damage process and the measured works of adhesion respectively

\begin{tabular}{|c|c|c|c|c|c|c|c|}
\hline \multirow[t]{2}{*}{ Composite } & \multirow{2}{*}{$\begin{array}{l}\text { Damage strain } \\
\text { rate }\left(\mathrm{s}^{-1}\right)\end{array}$} & \multirow[t]{2}{*}{$E_{O}(\mathrm{MPa})$} & \multicolumn{3}{|l|}{ Fitted quantities } & \multicolumn{2}{|c|}{ Inferred quantities } \\
\hline & & & $w_{o}\left(\mathrm{~J} \mathrm{~m}^{-3}\right)$ & $\varepsilon_{0}$ & $E_{L} / E_{O}$ at $\left(10^{-2} \mathrm{~s}^{-1}\right)$ & $E_{\text {dam }}(\mathrm{MPa})$ & $\begin{array}{l}\gamma(\dot{\varepsilon}) \\
\left(\mathrm{J} \mathrm{m}^{-2}\right)\end{array}$ \\
\hline QRX214 fine & $1 \times 10^{-2}$ & $6.4 \pm 0.3$ & $(20 \pm 3) \times 10^{4}$ & $0.35 \pm 0.02$ & $0.10 \pm 0.6$ & $1.6 \pm 0.3$ & $1.6 \pm 0.5$ \\
\hline QRX214 fine & $4 \times 10^{+3}$ & - & $(23 \pm 7) \times 10^{4}$ & $0.14 \pm 0.02$ & $0.48 \pm 0.03$ & $12 \pm 5$ & $1.9 \pm 0.5$ \\
\hline QRX217 coarse & $1 \times 10^{-2}$ & $7.8 \pm 0.5$ & $(4.7 \pm 0.6) \times 10^{4}$ & $0.14 \pm 0.01$ & $0.10 \pm 0.03$ & $2.4 \pm 0.5$ & $2.4 \pm 0.5$ \\
\hline QRX217 coarse & $4 \times 10^{+3}$ & - & $(4 \pm 1) \times 10^{4}$ & $0.06 \pm 0.01$ & $0.21 \pm 0.02$ & $11 \pm 5$ & $2.0 \pm 0.5$ \\
\hline
\end{tabular}

$\tan (\delta)$. In the case of coarse loaded composite, the value of $\gamma(\dot{\varepsilon})$ decreases with strain rate and there is no ready explanation, other than that it might be indicative a change in failure mechanism. One such candidate mechanism could be a change from partial to full debonding of the particles (until now we have only considered that the individual particles are either fully bonded or fully debonded).

The second inferred quantity is the modulus which is given by $\frac{w_{0}}{\varepsilon_{0}^{2}}$ and we have designated $E_{d a m}$. The physical interpretation of $E_{d a m}$ is an effective modulus local to the damage site, as damage is taking place. Note that the value of $E_{d a m}$ can be inferred separately for both the low rate of loading and the high rate loading; the latter is particularly interesting because it is typically the case that high rate moduli are experimentally difficult to obtain, due to concerns over sample force-equilibrium at low strains [24]. We note that the low rate value of $E_{d a m}$ is significantly less than the overall composite modulus at the same strain rate and that it increases by an order of magnitude at the higher strain rate. The latter is not surprising as polymer bonded explosives are known to stiffen with strain-rate [31] and the self-evident result of the data shown in Fig. 2.

\section{Conclusions and Outlook}

The major achievement of our study has been a clear experimental demonstration that the rate of compressive loading has a strong effect on damage evolution in RDXHTPB composite materials. Specifically we conclude that significantly less specific energy is required to inflict the same level of debonding damage at lower strain rates. This result has been shown quantitatively using simple, physically based, causal and damage metrics on well characterised materials, and as such these results form a good basis for the development and validation of models of the same.

We find that reasonable fits to the data under all conditions studied can be obtained using the Porter-Gould damage model and that a number of testable consequences of the model and associated relations are correctly captured. Specifically, the residual moduli fall as more mechanical energy is deposited into the samples (or equivalently the more they are strained), the composite with the coarse filler particles damages more readily than that with the fine fill, and substantially less energy is required to inflict the same level of damage at the lower strain-rate. However, in the case of the coarse grained composite, the data indicates that the damage activation energy (and by extension measured work of adhesion) has actually decreased with increasing strain rate. The finding is contrary to our expectations, and may indicate that for this material that there is a change in the physical nature of the damage mechanism(s) operative when the strain rate is increased. Presumably, any such change in damage mechanism is related to the increased mean particle size of the fill, which is the only substantial physical difference between the sample types. In order to take this framework of understanding forward, it would be useful to test whether there are in fact damage mechanisms occurring other than total debonding, and if so what are the relative significances. Candidate alternative mechanisms are partial (as opposed to total) debonding, binder rupture and filler particle cracking. It would also be useful to understand why the limiting moduli are greater at the higher strain rate, and whether or not this is an expected consequence of increased levels of inertial confinement.

We consider that the underlying behaviour should be quite generally applicable to particulate composites, whenever stiff filler particles are held in a viscoelastic matrix, and as such the general observations should be of wide utility.

Acknowledgments The authors wish to acknowledge financial support in the form of an Industrial CASE PhD Studentship for RLB funded by the UK Engineering and Physical Sciences Research Council (EPSRC) and by QinetiQ [EP/I501290/1]; UK MOD via a WSTC contract; DMW and APJ acknowledge the financial support of AWE. The data underlying this article can be found at the following persistent URL: https://www.repository.cam.ac.uk/handle/1810/253194. 
Open Access This article is distributed under the terms of the Creative Commons Attribution 4.0 International License (http://crea tivecommons.org/licenses/by/4.0/), which permits unrestricted use, distribution, and reproduction in any medium, provided you give appropriate credit to the original author(s) and the source, provide a link to the Creative Commons license, and indicate if changes were made.

\section{References}

1. Richter HP, Boyer LR, Graham KJ, Lepie AH, Zwierzchowski NG (1989) Shock sensitivity of damaged energetic materials. In: 9th Symposium (International) on detonation, Office of Naval Research publication number 113291-7, pp 1295-1309

2. Sandusky HW, and Bernecker RR (1993) Influence of fresh damage on the shock reactivity and sensitivity of several energetic materials. In: 10th International detonation symposium, Office of Naval Research publication number 33395-12, pp 490-498

3. Asay BW, Son SF, Bdzil JB (1996) The role of gas permeation in convective burning. Int J Multiph Flow 22:923-952

4. Berghout HL, Son SF, and Asay BW (2000) Convective burning in gaps of PBX 9501. In: International symposium on combustion, vol 28, pp 911-917

5. Palmer SJP, Field JE, Huntley JM (1993) Deformation, strengths and strains to failure of polymer bonded explosives. P R Soc A 440:399-419

6. Rae PJ, Goldrein HT, Palmer SJP, Field JE, Lewis AL (2002) Quasi-static studies of the deformation and failure of beta-HMX based polymer bonded explosives. P R Soc A 458:743-762

7. Williamson DM, Palmer SJP, Proud WG (2009) Brazilian disc testing of a UK PBX through the glass transition temperature. AIP Conf Proc 1195:560-563

8. Springer HK, Glascoe EA, Reaugh JE, Kercher JR, Maienschein JL (2012) Mesoscale modelling of deflagration-induced deconsolidation in polymer bonded explosives. AIP Conf Proc 1426:705-708

9. Dartois S, Nadot-Martin C, Halm D, Dragon A, Fanget A, Contesse G (2013) Micromechanical modelling of damage evolution in highly filled particulate composites-induced effects at different scales. Int J Damage Mech 22:927-966

10. Arora H, Tarleton E, Li-Mayer J, Charalambides MN, Lewis D (2015) Modelling the damage and deformation process in a plastic bonded explosive microstructure under tension using the finite element method. Comput Mater Sci 110:91-101

11. Cornish R, Porter D, Church P, Gould P, Andrews T, Proud B, Drodge D, Siviour C (2007) Comparison of Porter-Gould constitutive model with compression test data for HTPB/sugar. AIP Conf Proc 955:777-780

12. Murakami S (2012) Continuum damage mechanics. Springer, Dordrecht
13. Lawn BR, Wilshaw TR (1975) Fracture of brittle solids. Cambridge University Press, Cambridge

14. Nicholson DW (1979) On the detachment of a rigid inclusion from an elastic matrix. J Adhes 10:255-260

15. Gent AN (1980) Detachment of an elastic matrix from a rigid spherical inclusion. J Mater Sci 15:2884-2888

16. Siviour CR, Laity PR, Proud WG, Field JE, Porter D, Church PD, Gould PJ, Huntingdon-Thresher W (2008) High strain rate properties of a polymer-bonded sugar: their dependence on applied and internal constraints. P R Soc A 464:1229-1255

17. Gent AN, Schultz J (1972) Effect of wetting liquids on the strength of adhesion of viscoelastic materials. J Adhes 3:281-294

18. Maugis D, Barquins $M$ (1978) Fracture mechanics and the adherence of viscoelastic bodies. J Phys D 11:1989-2023

19. Johnson KL (1998) Mechanics of adhesion. Tribol Int 31:413-418

20. Porter D, Gould PJ (2009) Predictive nonlinear constitutive relations in polymers through loss history. Int J Solids Struct 46:1981-1993

21. Charrault E, Gauthier C, Marie P, Shirrer R (2009) Experimental and theoretical analysis of dynamic JKR contact. Langmuir 25:5847-5854

22. Siviour CR, (2005) High strain rate properties of materials using Hopkinson bar techniques. PhD Thesis, University of Cambridge

23. Pope PH, Field JE (1984) Determination of strain in a dynamic compression test. J Phys E 17:817-820

24. Gray III GT (2000) Classic split hopkinson bar testing. In: ASM handbook-mechanical testing and evaluation, vol 8, pp 463-476

25. Lea LJ, Jardine AP (2015) Two-wave photon Doppler velocimetry measurements in direct impact Hopkinson pressure bar experiments. EPJ Web Conf 94:01063

26. Drodge DR, Williamson DM (2016) Understanding damage in polymer bonded explosive composites. J Mater Sci 51:668-679

27. Boddy RL (2014) Damage in Energetic Materials. PhD Thesis, University of Cambridge

28. Trautmann A, Siviour CR, Walley SM, Field JE (2005) Lubrication of polycarbonate at cryogenic temperatures in the split Hopkinson pressure bar. Int J Impact Eng 31:532-544

29. Wiegand D, Reddingius B (2004) Mechanical properties of plastic bonded composites as a function of hydrostatic pressure. AIP Conf Proc 706:812-815

30. Hamilton NR, Williamson DM, Lewis D, Galuser A, and Jardine AP, (2015). Adhesive measurements of polymer bonded explosive constituents using the JKR experimental technique with a viscoelastic contact description. In: Proceedings of the 19th American Physical Society Shock Compression of Condensed Matter conference, Tampa, FL, 14-19 June 2015

31. Williamson DM, Siviour CR, Proud WG, Palmer SJP, Govier R, Ellis K, Blackwell P, Leppard C (2008) Temperature-time response of a polymer bonded explosive in compression (EDC37). J Phys D 41:085404 